\title{
Properties of disks and spiral arms along the Hubble sequence
}

\author{
J. $\mathrm{Ma}^{\star}$ \\ National Astronomical Observatories, Chinese Academy of Sciences, Beijing 100012, P.R. China \\ Received 6 December 2001 / Accepted 13 March 2002

\begin{abstract}
This paper presents some new statistical correlations between the properties of disks and of spiral arms with some physical properties of galaxies. Our results show that the thickness of spiral disks tends to diminish along the Hubble sequence in the sense that disks of Sc galaxies are $40 \%$ thinner than disks of Sab-Sb galaxies. Moreover, the thinner disks tend also to be bluer. We also find that there exists a correlation between HI linewidths and arm pattern within each Hubble type, which suggests that the arm shape is partially determined by the mass of a galaxy. Total mass luminosity ratios and total mass surface densities also have correlations with pitch angles i.e., for disks with lower surface densities and lower total mass luminosity ratios, the pitch angles tend to be greater.
\end{abstract}

Key words. galaxies: spiral - galaxies: structure

\section{Introduction}

When investigating the mass distribution within highly flattened, axisymmetric, self-gravitating spiral galaxies, Toomre (1963) found a family of exact solutions of the Poisson equation with arbitrary laws of rotation,

$\nabla^{2} \phi(R, z)=-4 \pi G \rho(R, z)=-4 \pi G \mu(R) \delta(z)$.

From Toomre's (1963) disk models of galaxies, Miyamoto \& Nagai (1975) introduced a new potential-density pair, in which the mass is no longer confined to an infinitesimally thin disk. This model is free of singularities and involves functions that are quite explicit and elementary.

Lin \& Shu $(1964,1966)$ did pioneering work to explain the spiral structure of disk galaxies using density waves to describe spiral patterns. These waves compress the gas component as it flows through the arms (Roberts et al. 1975). The spiral arms can also be the result of the tidal field of a nearby neighbor (Toomre \& Toomre 1972), bars (Sanders \& Huntley 1976) or Stochastic Self-propagating Star Formation (Gerola \& Seiden 1978; Mueller \& Arnett 1976).

Under the quasi-stationary spiral structure theory (QSSS) of density waves by Lin \& Shu (1964) and using the Green function method plus Fourier-Bessel transformation of three dimensional disk galaxies, Peng et al. $(1978,1979,1988)$ obtained the formulation of the gravitational potential of both galactic disks and spiral arms with different mathematical spiral curves. They generalized the Toomre models of two dimensional disk galaxies

\footnotetext{
* e-mail: majun@vega.bac.pku.edu.cn
}

to three dimensional models. That is to say, the distribution of surface density may be analytically derived from Toomre's rotation curves in a disk galaxy with a finite thickness. Based on the fundamental assumption (see details in Peng et al. 1979 or Peng 1988) that the density distribution along $z$-direction for a finite thickness disk is

$$
\rho(r, \phi, z)=\frac{1}{H} \sigma(r, \phi) \mathrm{e}^{-|z| / h},
$$

where $h$ is the vertical exponential scale height, $H=2 h$ the disk thickness and $\sigma(r, \phi)$ is the surface density, Peng et al. (1979) obtained a criterion for the appearance of density waves, which is

$r>r_{0}=\frac{H \sqrt{m^{2}+\Lambda^{2}}}{2}$

where $r_{0}$ is the polar coordinate of the starting point from which the arms of a galaxy stretch outward on the galactic plane, $m$ is the number of arms in a spiral galaxy, and $\Lambda$ is the winding parameter of the arm. Based on this criterion, Peng (1988) proposed a method for estimating the thickness of a non-edge-on spiral galaxy. Using this method, Ma et al. $(1997,1998)$ estimated the thickness of 72 spirals.

To study the component separation in edge-on spiral galaxies, van der Kruit \& Searle (1981a, 1981b, 1982a, $1982 \mathrm{~b}$ ) proposed a model for the three-dimensional distribution of light in galactic disks. Under the assumption that a disk galaxy has a locally isothermal, self-gravitating and truncated exponential disk, the space-luminosity of this model can be described by

$$
L(r, z)=L_{0} \mathrm{e}^{-r / h_{\mathrm{r}}} \operatorname{sech}^{2}\left(z / z_{0}\right) .
$$


A detailed investigation of edge-on spiral galaxies by de Grijs et al. (1997) showed, however, that the vertical light profiles are much closer to the exponential than to the isothermal solution.

The question of the mathematical form of spiral arms was recognized at the beginning of this century (von der Pahlen 1911; Groot 1925). Then, Danver (1942), Kennicutt (1981) and Kennicutt \& Hodge (1982) systematically studied the shapes of spiral arms by fitting different spiral curves by least squares and using an iterative procedure. These authors concluded that spiral arms are better represented by logarithmic spirals.

There are many determinations of the pitch angles of spiral arms that can be found in the literature. Danver (1942) measured these values for a sample of 98 nearby spirals. Kennicutt (1981) measured the shapes of spiral arms in 113 nearby Sa-Sc galaxies. Using the Fourier analysis first proposed by Kalnajs (1975) and further developed by Considère \& Athanassoula (1982, 1988), García-Gómez \& Athanassoula (1993) calculated the pitch angles in a sample of 44 spiral galaxies. Puerari \& Dottori (1992) using also a similar technique measured the pitch angles in 22 galaxies. Recently, Seigar \& James (1998a, 1998b) determined the pitch angles of spiral arms for 45 spiral galaxies based on the images in the near-IR $J$ and $K$ bands. With the IRAF software, Ma et al. $(1997,1998)$ directly fitted the shapes of spiral arms on the images of 72 northern spiral galaxies. The details of this procedure can be found in Ma et al. (1997, 1998, 1999). These measures on the pitch angles can be used to understand the influence of the different physical properties of galaxy disks on arm shapes.

The structure of this paper is as follows: in Sect. 2 we present a correlation between galaxy type or color and disk thickness. In Sect. 3, we show some new correlations between the pitch angles of spiral arms and the physical properties of galaxy disks. Finally, in Sect. 4 we present our conclusions.

\section{Statistical properties of disk thickness along the Hubble sequence}

Our statistical sample contains 72 northern spiral galaxies, for which the values of disk thickness, inclination and pitch angle of individual spiral arms were measured in Ma et al. (1997, 1998). A more detailed discussion can be found in Ma et al. (1999).

Ma et al. $(1998,1999)$ have presented some statistical correlations between thickness and flatness of spiral disks with some other physical properties of spiral galaxies. The main conclusions are that the thickness is correlated with color, mass in neutral hydrogen and $\mathrm{H}_{\alpha}+[\mathrm{NII}]$ equivalent width (see details in Ma et al. 1998, 1999). In this section, we will investigate a new correlation between thickness or flatness and total luminosity.

Roberts \& Haynes (1994) systematically studied the physical parameters along the Hubble sequence by making use of two primary catalogues the first one is the RC3

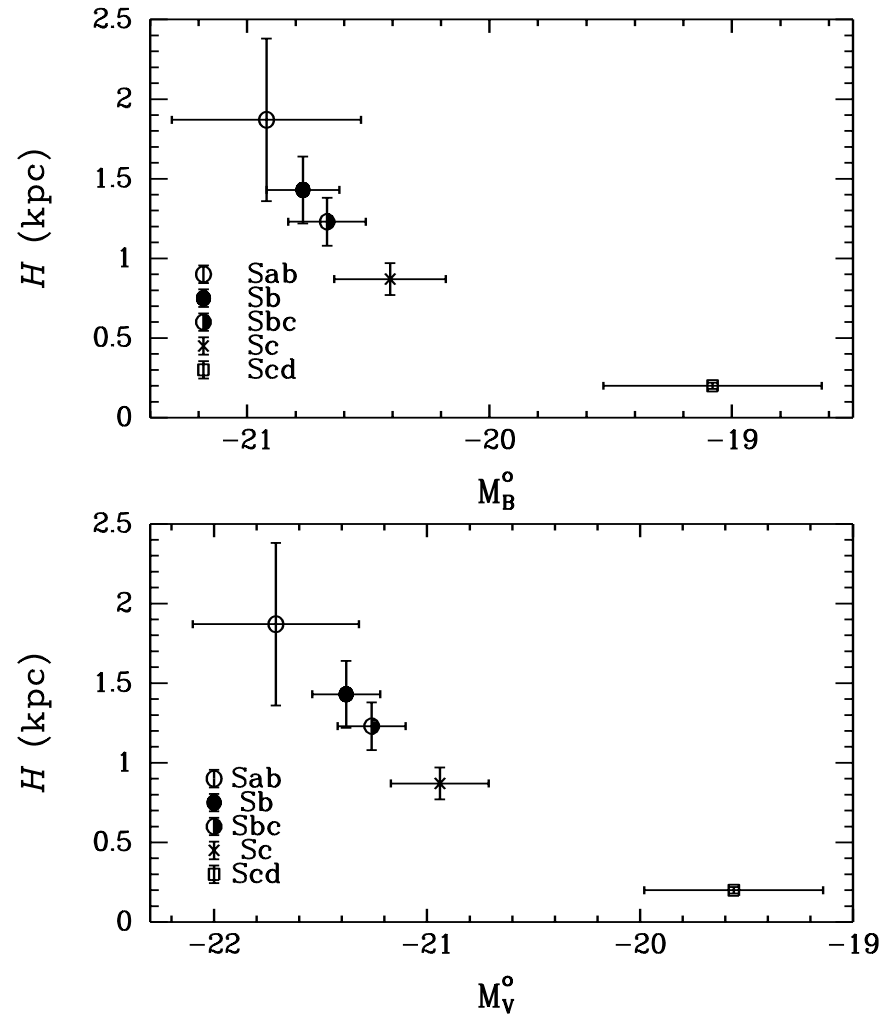

Fig. 1. Mean thickness of spiral galaxy disks plotted against the mean total ("face-on") absolute magnitude in the $B$ and $V$ systems for each Hubble type of the galaxies in our sample. The upper panel shows this relation for the $B$ magnitudes while the lower panel shows this relation for the $V$ magnitudes.

catalogue (de Vaucouleurs et al. 1991) and the second one is a private catalogue maintained by R. Giovanelli \& M. Haynes. These authors showed that the mean total luminosity of the galaxies changes only slightly as a function of Hubble type until the latest types (Scd). The mean total luminosity, however, clearly shows a decrease for the dwarf galaxies (Sd and $\mathrm{Sm}$ ).

In order to study the correlation between thickness along the Hubble sequence and total ("face-on") absolute magnitude from the RC3 catalogue (de Vaucouleurs et al. 1991), we plot in Fig. 1 the mean thickness for each Hubble type of the galaxies in our sample versus mean total luminosity. In this figure a clear trend is shown, namely, galaxies of later Hubble types tend to be flatter than galaxies of early types. More specifically, Scd galaxies are, on average, $40 \%$ flatter than $\mathrm{Sb}$ galaxies. The total absolute optical magnitudes in the $B$ and $V$ systems, which are corrected for differential galactic and internal extinction (to "face-on"), and the Hubble types, come from the RC3 catalogue (de Vaucouleurs et al. 1991). We calculated the mean and dispersion of the distributions of $H$ between different Hubble types. Table 1 lists the relevant quantities for these distributions. In Col. 1 we give the Hubble type and in Col. 3 the numbers of galaxies in each Hubble type, as well as the difference. Column 4 gives the means of the distributions of $H$, Col. 5 the dispersion around the means. In Col. 6 we present the probability that the two 
Table 1. Parameters of the distribution of thicknesses in the different Hubble type.

\begin{tabular}{cccccc}
\hline \hline Hubble type & & Number of points & Mean & Dispersion & t-Std. \\
\hline Sab and Sb & & 18 & 1.53 & 0.19 & \\
Sbc & & 24 & 1.23 & 0.15 & 0.05 \\
& Difference & 6 & 0.30 & 0.04 & \\
\hline Sab and Sb & & 18 & 1.53 & 0.19 & \\
$\mathrm{Sc}$ & & 21 & 0.87 & 0.10 & 0.20 \\
& Difference & 3 & 0.66 & 0.09 & \\
\hline $\mathrm{Sbc}$ & & 24 & 1.23 & 0.15 & \\
$\mathrm{Sc}$ & & 21 & 0.87 & 0.10 & 0.07 \\
& Difference & 3 & 0.36 & 0.05 & \\
\hline $\mathrm{Sc}$ & & 21 & 0.87 & 0.10 & \\
$\mathrm{Scd}$ & & 4 & 0.20 & 0.02 & 0.05 \\
& Difference & 17 & 0.67 & 0.08 & \\
\hline
\end{tabular}

$H$ means between different Hubble types come from the same distribution. Since there are only 4 Sab galaxies in this study, we merge these galaxies with the Sb galaxies to get a single Sab-Sb class. From the Student's $t$-test we can see that the means of the distributions of $H$ between different Hubble types are different, except for the means between the Sab-Sb class and Sc class.

Guthrie (1992) derived the axial ratios of disk components, $R$, for 262 edge-on spiral galaxies, and analyzed the distribution of isophotal axial ratios for 888 diameterlimited normal Sa-Sc galaxies to obtain information on the true axial ratios $R_{0}$. If we suppose that the radial to vertical scale parameter ratio is equal to $R_{0}$ from Guthrie (1992), we can calculate the values of $\overline{\log R_{0}}$ for each Hubble type of the galaxies in our sample.

Figure 2 shows the dependence of $\overline{\log R_{0}}$ on galaxy type in the RC3 catalogue (de Vaucouleurs et al. 1991). In this figure we can see a correlation between $\overline{\log R_{0}}$ and Hubble sequence, in the sense that galaxies become systematically flatter when going from type Sab to Scd. We also calculated the mean and dispersion of the distribution of $\overline{\log R_{0}}$ between different Hubble types. Table 2 summarises the relevant quantities of this distribution as in Table 1. We also merge Sab galaxies with the Sb galaxies to get a single Sab-Sb class. From the Student's $t$-test we can see that the means of the distributions of $\overline{\log R_{0}}$ between different Hubble types are also different except for the means between Sab-Sb class and Sbc class.

Ma et al. (1998) have studied the correlation between thickness and color index $\left((B-V)_{\mathrm{T}}^{0}\right)$ between the $B$ and $V$ bands, and presented the conclusion that thinner spirals are bluer. Figure 3 plots the correlation of average thickness with color index $(B-V)_{\mathrm{T}}^{0}$ along the Hubble type in the RC3 catalogue (de Vaucouleurs et al. 1991). The color indices, which are corrected for differential galactic and internal extinction (to "face-on"), and for redshift between the $B$ and $V$ bands, are also taken from the RC3 catalogue (de Vaucouleurs et al. 1991). The corresponding averages for each Hubble type are showed in Fig. 3 as in Fig. 1. Figure 3 shows that the correlation between average thickness and color index is also monotonic. Namely, along the Hubble sequence the galaxies tend to be bluer

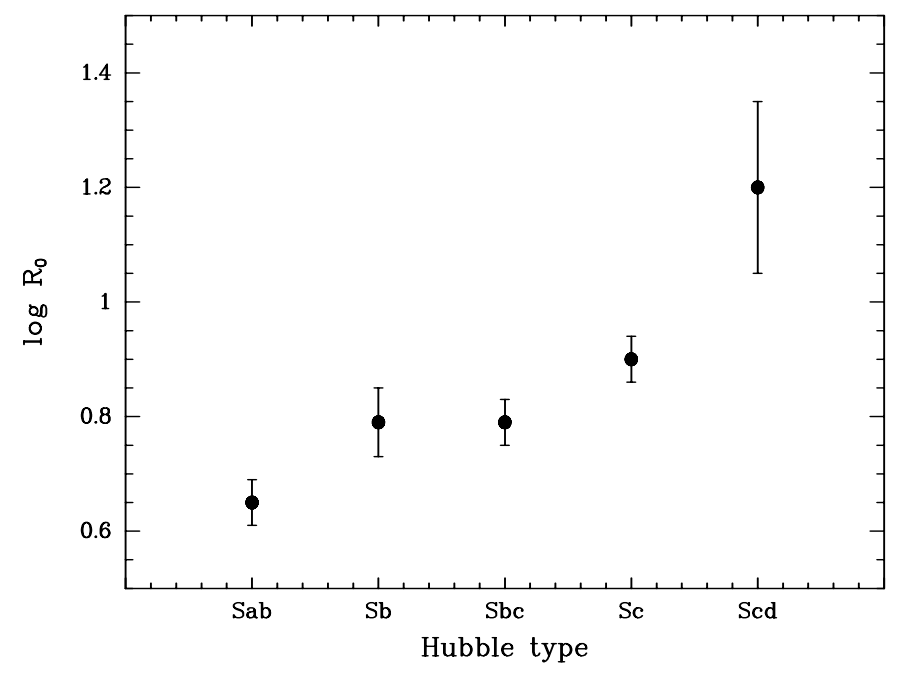

Fig. 2. Dependence of $\overline{\log R_{0}}$ on galaxy type in RC3.

and thinner. We also calculated the mean and dispersion of the distribution of $(B-V)_{\mathrm{T}}^{0}$ between different Hubble types. Table 3 summarises some of the relevant quantities for this distribution as in Tables 1 and 2. We also merge $\mathrm{Sab}$ galaxies with the Sb galaxies to get a single Sab-Sb class. From the Student's $t$-test we can see that the means of the distributions of $(B-V)_{\mathrm{T}}^{0}$ between different Hubble types are not different except for the means between the Sc class and Scd class.

\section{Statistical properties of spiral arms with some physical properties of galaxies}

\subsection{Comparison of our pitch angle values with other sources}

In this section, we will show some new correlations between the properties of spiral arms and some physical properties of galaxy disks. But, first, it will be useful to compare the pitch angles of the common galaxies, which were measured by Danver (1942), Kennicutt (1981) or other authors (Considère \& Athanassoula 1988; Puerari \& Dottori 1992; García-Gómez \& Athanassoula 1993). The comparisons are plotted in Fig. 4. In this figure, 
Table 2. Parameters of the distribution of $\overline{\log R_{0}}$ in the different Hubble type.

\begin{tabular}{cccccc}
\hline \hline Hubble type & & Number of points & Mean & Dispersion & t-Std. \\
\hline Sab and Sb & & 20 & 0.75 & 0.04 & \\
$\mathrm{Sbc}$ & 24 & 0.79 & 0.04 & 0.25 \\
& Difference & 4 & 0.04 & 0.00 & \\
\hline $\mathrm{Sbc}$ & & 24 & 0.79 & 0.04 & \\
$\mathrm{Sc}$ & & 22 & 0.90 & 0.04 & 0.07 \\
& Difference & 2 & 0.11 & 0.00 & \\
\hline $\mathrm{Sc}$ & & 22 & 0.90 & 0.04 & \\
$\mathrm{Scd}$ & & 6 & 1.20 & 0.15 & 0.06 \\
& Difference & 16 & 0.30 & 0.11 & \\
\hline
\end{tabular}

Table 3. Parameters of the distribution of $B-V$ color in the different Hubble type.

\begin{tabular}{cccccc}
\hline \hline Hubble type & & Number of points & Mean & Dispersion & t-Std. \\
\hline Sab and Sb & & 18 & 0.65 & 0.03 & \\
Sbc & & 24 & 0.59 & 0.01 & 0.61 \\
& Difference & 6 & 0.06 & 0.02 & \\
\hline Sab and Sb & & 18 & 0.65 & 0.03 & \\
$\mathrm{Sc}$ & & 21 & 0.53 & 0.02 & 0.92 \\
& Difference & 3 & 0.12 & 0.01 & \\
\hline $\mathrm{Sbc}$ & & 24 & 0.59 & 0.01 & \\
$\mathrm{Sc}$ & & 21 & 0.53 & 0.02 & 0.76 \\
& Difference & 3 & 0.06 & 0.01 & \\
\hline $\mathrm{Sc}$ & & 21 & 0.53 & 0.02 & \\
$\mathrm{Scd}$ & & 4 & 0.47 & 0.04 & 0.13 \\
& Difference & 17 & 0.06 & 0.02 & \\
\hline
\end{tabular}

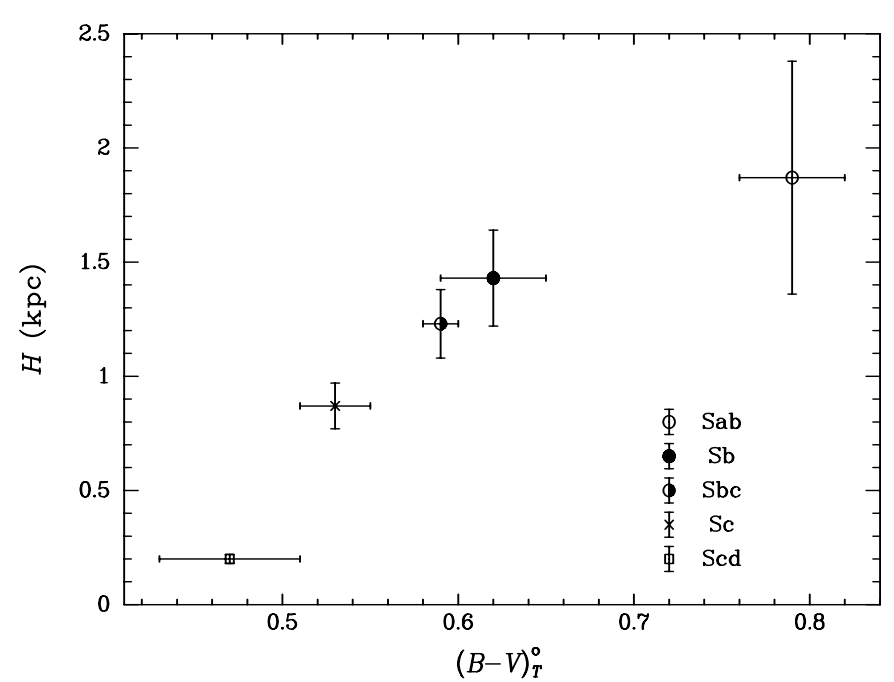

Fig. 3. Thickness of spiral galaxy disks plotted against the corrected $B-V$ color. Both thickness and color are the corresponding means for each Hubble type of the galaxies in our sample.

the dashed lines are the diagonal, and the solid lines are the result of the linear correlations. For each line, we calculated the correlation coefficient, which is shown in Table 4, together with the number of points used for each correlation. In Col. 1 we list the symbol of panel in Fig. 4 and in Col. 2 the number of galaxies. Column 3 gives the linear correlation coefficient. From Table 4 and Fig. 4, we can see that our data correlate better with the
Table 4. Linear correlation coefficients.

\begin{tabular}{ccc}
\hline \hline Fig. 4 & Number of points & Correlation coefficient \\
\hline a) & 35 & 0.71 \\
b) & 21 & 0.74 \\
c) & 20 & 0.63 \\
d) & 13 & 0.69 \\
\hline
\end{tabular}

values of Danver and Kennicutt than the data coming from the other sources. The reason may be that our method is similar to the method used by Danver and Kennicutt.

\subsection{Pitch angles as a function of Hubble type}

Hubble $(1926,1936)$ introduced a scheme to classify galaxies that is still in use today. Galaxy types run from elliptical, to lenticular, spiral and finally, irregular galaxies. This scheme, which was extended by some astronomers (Holmberg 1958; de Vaucouleurs 1956, 1959; Morgan 1958, 1959; van den Bergh 1960a,b, 1976; Sandage 1961; Sandage \& Tammann 1981, 1987; Sandage \& Bedke 1993), is based on a number of observational criteria such as the gas content, size of nuclear bulges, resolution of the spiral arms, and pitch angle of spiral arms.

The tightness of spiral arms, in addition to the degree of resolution in the arm and the relative size of the unresolved nuclear region, are the fundamental criteria in Hubble's classification of spiral galaxies (Hubble 1926, 1936). In order to better understand the nature and 

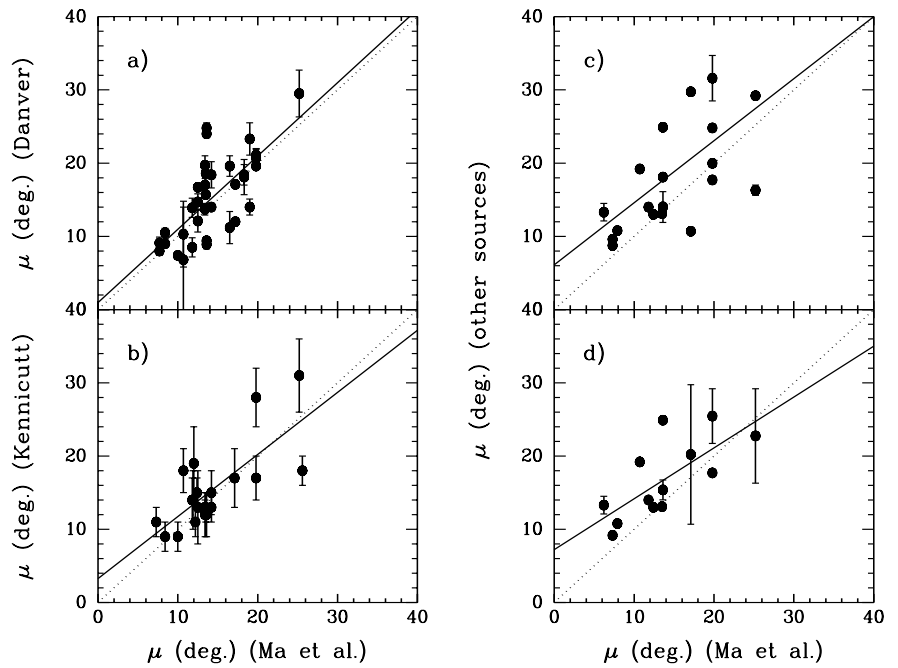

Fig. 4. Comparison of our pitch angle results (Ma et al. 1997, 1998) with a) Danver (1942), b) Kennicutt (1981), and c) Considère \& Athanassoula (1988), Puerari \& Dottori (1992), and García-Gómez \& Athanassoula (1993), d) mean pitch angles from the sources of $\mathbf{c}$ ), for the galaxies in common. In the panel of $\mathbf{d}$ ), the vertical error bars are the rms calculated from all values in the sources of $\mathbf{c}$ ).

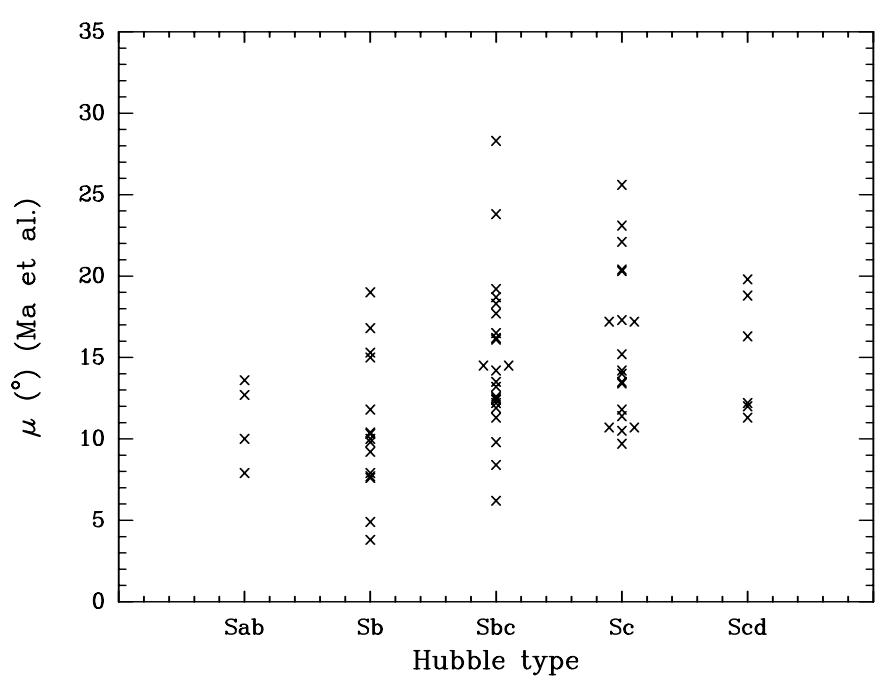

Fig. 5. Mean measured pitch angle plotted against different Hubble types in RC3.

origin of the Hubble sequence and evaluate the difference in the classification, Kennicutt (1981) compared his measures of pitch angles with the Hubble type as determined by Sandage \& Tammann (1981, hereafter ST) and Yerkes class by Morgan (1958, 1959). From Figs. 7 and 8 in Kennicutt (1981), we can see that, although ST's classification is based almost solely upon disk resolution and Morgan's solely on the central concentration of the galaxies, the trends between arm pitch angles and the galaxy types in both classifications are almost the same. In the classifications of galaxies by de Vaucouleurs et al. (1976, RC2; 1991, RC3), the three criteria are considered.
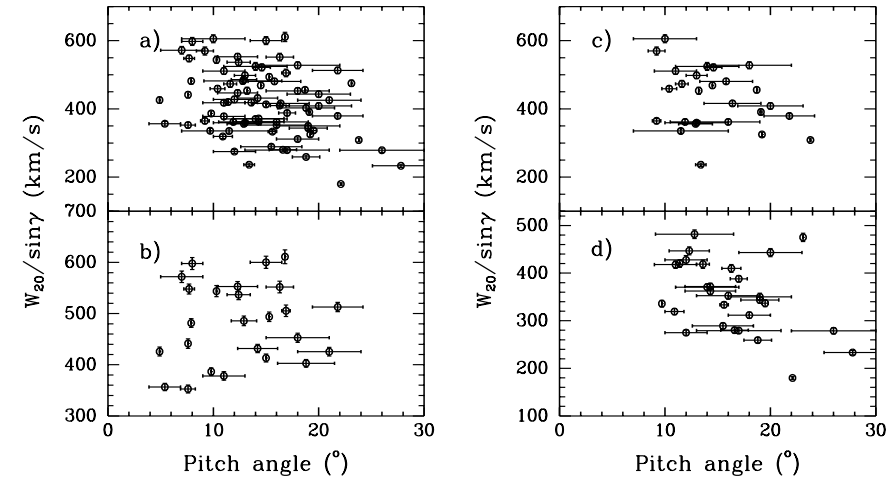

Fig. 6. HI linewidths plotted against measured pitch angle, a) all Hubble types, b) Sab and Sb, c) Sbc,d) Sc and Scd.

To clearly see the weight of the tightness of spiral patterns in the RC3 catalogue (de Vaucouleurs et al. 1991), we plot the measured pitch angles by Ma et al. (1997, 1998) against the Hubble types coming from the RC3 catalogue (de Vaucouleurs et al. 1991) in Fig. 5. We can see that there is an increase in the mean pitch angles along the Hubble sequence. This trend represents the spirit of Hubble's original scheme for the classification of spirals. This relation has been discussed by many authors such as Kennicutt (1981), Considère \& Athanassoula (1988), Puerari \& Dottori (1992), and García-Gómez \& Athanassoula (1993). Our measures on the pitch angles follow the expected relation with Hubble type as for the rest of authors. Note, however, that the dispersions for each Hubble type are very large. For some Sab galaxies the pitch angles can be larger than for some Scd galaxies. So, it is obvious that the weight of tightness for spiral patterns in the RC3 catalogue (de Vaucouleurs et al. 1991) is only qualitative, not quantitative.

\subsection{HI linewidths vs. pitch angle}

Tully \& Fisher (1977) studied the correlation between distance-independent observable, global neutral hydrogen line profile widths and absolute magnitude or diameter of spiral galaxies. Using these measures, they offered a tool for measuring extragalactic distance, as well as a method of estimating the Hubble constant. In this study, we will present some statistical properties of spiral arms with some physical properties of galaxies using the weighted mean HI profile linewidths defined at the level of $20 \%$ of the peak flux (called $W_{20}$ ) from the RC3 catalogue (de Vaucouleurs et al. 1991). These values are corrected for bandwidth, but not for redshift. The inclinations are derived by Tully's formula (1988)

$$
\gamma=\arccos \sqrt{\left[\left(\frac{d_{25}}{D_{25}}\right)^{2}-0.2^{2}\right] /\left(1-0.2^{2}\right)}+3^{\circ},
$$

where $D_{25}$ and $d_{25}$, which are the apparent major and minor isophotal diameters measured at, or reduced down to the surface brightness level $\mu_{B}=25.0 \mathrm{~B}$ magnitudes per square arcsecond, come from the RC3 catalogue 
Table 5. Spearman rank-order correlation coefficients.

\begin{tabular}{cccc}
\hline \hline Fig. 6 & Number of points & Correlation coefficient & Significance \\
\hline a) & 82 & -0.34 & 0.0016 \\
b) & 25 & 0.15 & 0.48 \\
c) & 27 & -0.35 & 0.071 \\
d) & 30 & -0.40 & 0.029 \\
\hline
\end{tabular}

(de Vaucouleurs et al. 1991). The inclinations $(\gamma)$ of all these galaxies exceed $50^{\circ}$ from face-on so that there is no appreciable error in correcting the hydrogen profile for projection. In Fig. 6, we plot the correlation between pitch angle and $W_{20}$. The pitch angles are from Danver (1942), Kennicutt (1981), Ma et al. (1997, 1998) and other authors (Considère \& Athanassoula 1988; Puerari \& Dottori 1992; García-Gómez \& Athanassoula 1993). The horizontal error bars are from these sources, or the rms calculated from all values in the literature if there are different values of the pitch angle for some particular galaxy. In this figure, we do not include the galaxies for which the values of the rms of the pitch angles are larger than 5 degrees. Panel a) shows the correlation pooling together all the data, b) the data for Sab and Sb galaxies, c) the data for Sbc galaxies and d) the data for Sc and Scd galaxies. The panel a) shows that there exits a correlation between arm shape and $W_{20}$, which has been presented by Kennicutt \& Hodge (1982). However, the data show a great dispersion. Besides, as HI linewidths and pitch angles both are somewhat related through Hubble types, this panel can be dominated by the effect of the Hubble sequence. We may not conclude from this panel alone that the pitch angles are related to the $W_{20}$. In order to eliminate the effect of the Hubble sequence, we show the panels b), c) and d) separating the galaxies into their respective Hubble types. For each group, we calculated the Spearman rank-order correlation coefficient and its significance. Table 5 summarises the relevant quantities. In Col. 1 we list the symbol of the panel in Fig. 6 and in Col. 2 the number of data used for each panel. Column 3 gives the correlation coefficient and Col. 4 its significance. From Fig. 6 and Table 5, we can see that, except for the case of Sab-Sb galaxies, there exists a correlation between the arm shape and $W_{20}$ for each Hubble type separately. The case of panel b) can be due in part to the difficulties in determining the HI line widths for these galaxies, due to the lower HI contents, specially in the central parts. However, it is clear that $W_{20}$ cannot be the only parameter that dictates arm shape, since the scatter is large.

As we know, more massive galaxies have more stars than less massive ones, and more massive galaxies rotate faster. $W_{20}$ connects with the maximum rotation velocity of a galaxy. Tully \& Fouqué (1985) proposed a formula that could transform the $20 \%$ linewidths into the parameter $W_{R}$, which approximates twice the maximum rotation velocity of a galaxy. The correlation between pitch angle and $W_{20}$ can indicate that the arm shape of spiral galaxies is partially determined by the mass of a galaxy.

\subsection{Total mass luminosity ratio and total mass surface density vs. pitch angle}

Roberts \& Haynes (1994) systematically studied the physical parameters along the Hubble sequence. They showed that the total mass luminosity ratios $\left(M_{\mathrm{T}} / L_{B}\right)$ of spiral galaxies are nearly independent of Hubble type, and that the total mass surface densities $\left(\sigma_{\mathrm{T}}\right)$ are also approximately constant along the Hubble sequence for Sab to Sc galaxies, showing some decrease for Scd galaxies. Figure 7 shows the correlations between pitch angle and total mass luminosity ratio (panel a), and total mass surface density (panel b). The sources of pitch angles and horizontal error bars are the same as in Fig. 6. The total mass luminosity ratios and total mass surface densities are calculated as in Roberts \& Haynes (1994) using the RC3 catalogue (de Vaucouleurs et al. 1991). In this figure, the inclinations $(\gamma)$ of all these galaxies also exceed $50^{\circ}$ from face-on so that there is no appreciable error in correcting the hydrogen profile for projection. For simplicity, as in Roberts \& Haynes (1994), we use the corrected $20 \%$ linewidths as the measure of $2 V_{\text {rot }}$ and $D_{25}$ as the indicator of $2 R$. For each correlation, we calculated the Spearman rank-order correlation coefficient and its significance. Table 6 summarises some of the relevant quantities. In Col. 1 we list the symbol of the corresponding panel in Fig. 7 and in Col. 2 the number of galaxies. Column 3 gives the correlation coefficient and Col. 4 its significance. From Fig. 7 and Table 6 , we can see a significant trend in the sense that for lower total mass luminosity ratio and lower mass surface density the pitch angles tend to be larger, although the scatter in this figure is also large.

\section{Conclusion}

In this paper, we investigate some new statistical correlations between the physical properties of disks and of spiral arms with some physical properties of galaxies. Our main conclusions are: (1). Along the Hubble sequence, the spiral galaxies tend to be thinner and somewhat bluer, specially the later types, Sc-Scd. (2). For later Hubble types, there is a correlation between arm pitch angle and HI linewidths. This correlation exits for each Hubble type separately and can indicate that the arm shape is partially determined by the mass of the galaxy. (3). As the pitch angles increase, the total mass luminosity ratios and total mass surface densities decrease. 
Table 6. Spearman rank-order correlation coefficients.

\begin{tabular}{cccc}
\hline \hline Fig. 7 & Number of points & Correlation coefficient & Significance \\
\hline a) & 82 & -0.22 & 0.050 \\
b) & 82 & -0.24 & 0.038 \\
\hline
\end{tabular}

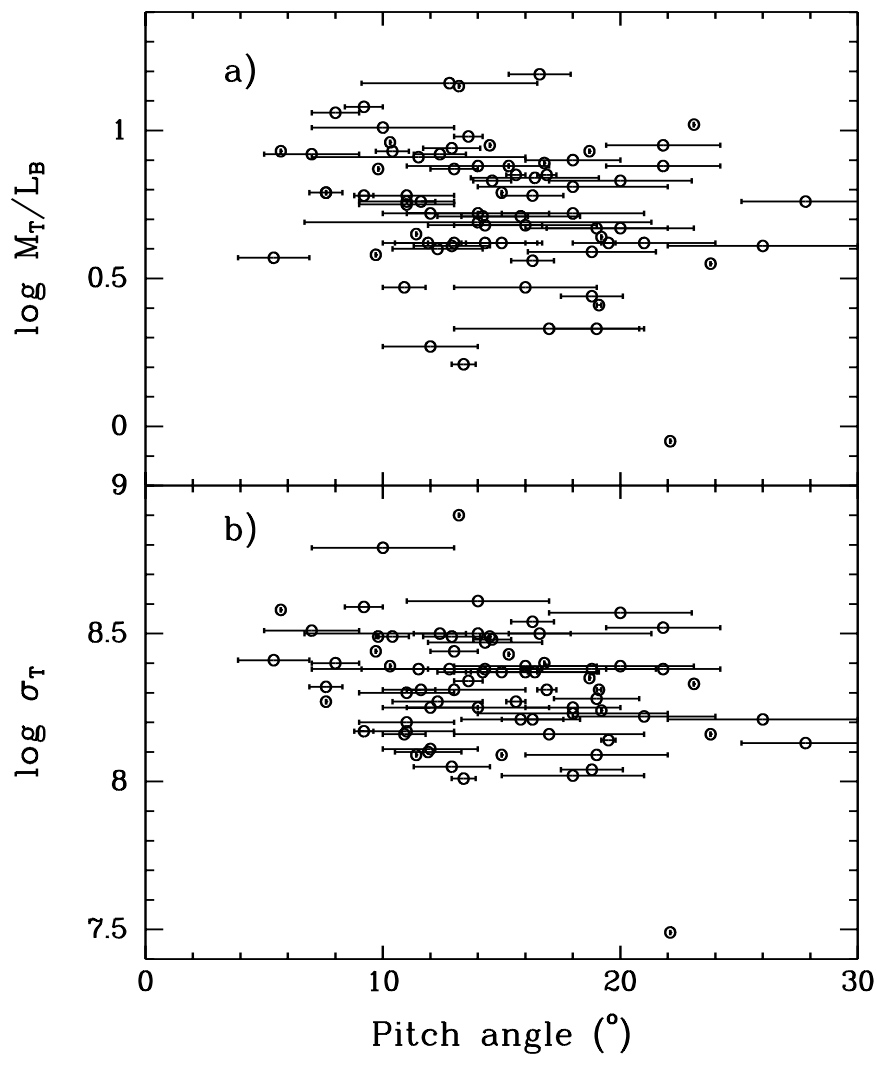

Fig. 7. Total mass luminosity ratio and total mass surface density plotted against measured pitch angle.

Acknowledgements. We are indebted to the referee, Dr. Carlos García-Gómez, for many critical comments and helpful suggestions, and for English editing that have greatly improved our paper.

\section{References}

Considère, S., \& Athanassoula, E. 1982, A\&A, 111, 28 Considère, S., \& Athanassoula, E. 1988, A\&AS, 76, 365 Danver, C. C. 1942, Ann. Obs. Lund., 10

de Vaucouleurs, G. 1956, Mem. Commonwealth Obs. (Mount Stromolo), 3(13)

de Vaucouleurs, G. 1959, Handbuch der Physik, 53, 275

de Vaucouleurs, G., de Vaucouleurs, A., \& Corwin, H. G. Jr 1976, The Second Reference Catalogue of Bright Galaxies (Austin: University of Texas) (RC2)

de Vaucouleurs, G., de Vaucouleurs, A., Corwin, H. G. Jr, et al. 1991, The Third Reference Catalogue of Bright Galaxies (New York: Springer-Verlag) (RC3)

de Grijs, R., Peletier, R. F., \& van der Kruit, P. C. 1997, A\&A, 327,966

Gerola, H., \& Seiden, P. E. 1978, ApJ, 223, 129

García-Gómez, C., \& Athanassoula, E. 1993, A\&AS, 100, 431
Groot, H. 1925, MNRAS LXXXV, 6, 525

Guthrie, B. N. G. 1992, A\&AS, 93, 255

Holmberg, E. 1958, Medd. Lund. Obs. Ser., 2(136)

Hubble, E. P. 1926, ApJ, 64, 321

Hubble, E. P. 1936, The Realm of the Nebuloe (New Haven: Yale Univ. Press)

Kalnajs, A. J. 1975, La dynamique des galaxies spirales, ed. L. Weliachew, Colloq. Internat. CNRS, 241, 103

Kennicutt, R. C. 1981, AJ, 86, 1847

Kennicutt, R. C., \& Hodge, P. 1982, ApJ, 253, 101

Lin, C. C., \& Shu, F. H. 1964, ApJ, 140646

Lin, C. C., \& Shu, F. H. 1966, Proc. Nat. Acad. Sci., 55, 229

Ma, J., Peng, Q. H., \& Gu, Q. S. 1997, ApJ, 490, L51

Ma, J., Peng, Q. H., \& Gu, Q. S. 1998, A\&AS, 130, 449

Ma, J., Zhao, J. L., Shu, C. G., et al. 1999, A\&A, 350, 31

Miyamoto, M., \& Nagai, R. 1975, PASJ, 27, 533

Morgan, W. W., PASP 1958, 70, 364

Morgan, W. W., PASP 1959, 71, 394

Mueller, M. W., \& Arnett, W. D. 1976, ApJ, 210, 670

Peng, Q. H., Huang, K. L., \& Huang, J. H. 1978, Acta. Astron. Sin., 19, 182

Peng, Q. H., Li, X. Q., Huang, K. L., et al. 1979, Sci. China XXII, 925

Peng, Q. H. 1988, A\&A, 206, 18

Puerari, I., \& Dottori, H. A. 1992, A\&AS, 93, 469

Roberts, W. W., Roberts, M. S., \& Shu, F. H. 1975, ApJ, 196, 381

Roberts, M. S., \& Haynes, M. P. 1994, ARA\&A, 32, 115

Sandage, A. R. 1961, The Hubble Atlas of Galaxies (Washington: Carnegie Institute of Washington)

Sandage, A. R., \& Tammann, G. A. 1981, A Revised ShapleyAmes Catalog of Galaxies (Washington: Carnegie Institute of Washington)

Sandage, A. R., \& Tammann, G. A. 1987, A Revised ShapleyAmes Catalog of Bright Galaxies (Washington: Carnegie Institute of Washington)

Sandage, A. R., \& Bedke, J. 1993, Carnegie Atlas of Galaxies (Washington: Carnegie Institute of Washington)

Sanders, R. H., \& Huntley, J. M. 1976, ApJ, 209, 53

Seigar, M. S., \& James, P. A. 1998a, MNRAS, 299, 672

Seigar, M. S., \& James, P. A. 1998b, MNRAS, 299, 685

Toomre, A. 1963, ApJ, 138, 385

Toomre, A., \& Toomre, J. 1972, ApJ, 178, 623

Tully, R. B., \& Fisher, J. R. 1977, A\&A, 54, 661

Tully, R. B., \& Fouqué, P. 1985, ApJS, 58, 67

Tully, R. B. 1988, Nearby Galaxies Catalogue (Cambridge: Cambridge Univ. Press)

van der Kruit, P. C., \& Searle, L. 1981a, A\&A, 95, 105 van der Kruit, P. C., \& Searle, L. 1981b, A\&A, 95, 116 van der Kruit, P. C., \& Searle, L. 1982a, A\&A, 110, 61 van der Kruit, P. C., \& Searle, L. 1982b, A\&A, 110, 79 van den Bergh, S. 1960a, ApJ, 131, 215

van den Bergh, S. 1960b, ApJ, 131, 558

van den Bergh, S. 1976, ApJ, 206, 883

von der Pahlen, E. 1911, AN, 188, 249 